\title{
FINITELY GENERATED SUBMODULES OF DIFFERENTIABLE FUNCTIONS
}

\author{
B. ROTH
}

\begin{abstract}
Suppose $\left[\mathscr{E}^{m}(\Omega)\right]^{p}$ is the Cartesian product of the space of real-valued $m$-times continuously differentiable functions on an open set $\Omega$ in $R^{n}$ with itself $p$-times where $m$ is finite and $\Omega$ is connected. $\left[\mathscr{E}^{m}(\Omega)\right]^{p}$ is a $\mathscr{E}^{m}(\Omega)$-module. The finitely generated submodules of $\left[\mathscr{E}^{m}(\Omega)\right]^{p}$ are $\operatorname{im}(F)$ where $F:\left[\mathscr{E}^{m}(\Omega)\right]^{a} \rightarrow\left[\mathscr{E}^{m}(\Omega)\right]^{p}$ is a $p \times q$ matrix $\left(f_{i j}\right)_{1 \leqq i \leqq p, 1 \leqq j \leqq q}, f_{i j} \in \mathscr{E}^{m}(\Omega)$. In the present paper, it is shown that $\operatorname{im}(F)$ is closed in $\left[\mathscr{E}^{m}(\Omega)\right]^{p}$ if and only if the rank of the matrix $\left(f_{i j}(x)\right)_{1 \leqq i \leqq p, 1 \leqq j \leqq q}$ is constant for $x \in \Omega$. Applications are made to systems of division problems for distributions.
\end{abstract}

1. Introduction. Let $\mathscr{E}^{m}(\Omega)$ denote the algebra of real-valued $m$-times continuously differentiable functions on an open set $\Omega$ in $R^{n}$ equipped with the topology of uniform convergence of all derivatives of order $\leqq m$ on all compact subsets of $\Omega$. Throughout we assume that $m$ is finite and $\Omega$ is connected.

Let $\left[\mathscr{E}^{m}(\Omega)\right]^{p}$ denote the Cartesian product of $\mathscr{E}^{m}(\Omega)$ with itself $p$-times equipped with the product topology. Then $\left[\mathscr{E}^{m}(\Omega)\right]^{p}$ is a Fréchet space and a $\mathscr{E}^{m}(\Omega)$-module. We here characterize the finitely generated submodules of $\left[\mathscr{E}^{m}(\Omega)\right]^{p}$ which are closed in $\left[\mathscr{E}^{m}(\Omega)\right]^{p}$. Interest in closed finitely generated submodules of $\left[\mathscr{E}^{m}(\Omega)\right]^{p}$ stems primarily from their connection by duality with systems of division problems for distributions (see §3).

2. The main result. Consider the finitely generated submodule $M=$ $\left\{g_{1} \mathbf{f}_{1}+\cdots+g_{q} \mathbf{f}_{q}: g_{1}, \cdots, g_{q} \in \mathscr{E}^{m}(\Omega)\right\}$ of $\left[\mathscr{E}^{m}(\Omega)\right]^{p}$ where $\mathbf{f}_{j}=\left(f_{1_{j}}, \cdots, f_{p_{j}}\right) \in$ $\left[\mathscr{E}^{m}(\Omega)\right]^{p}$ for $1 \leqq j \leqq q$. Let $F$ be the $p \times q$ matrix $\left(f_{i j}\right)_{1 \leqq i \leqq p, 1 \leqq j \leqq q}$. Then $F:\left[\mathscr{E}^{m}(\Omega)\right]^{q} \rightarrow\left[\mathscr{E}^{m}(\Omega)\right]^{p}$ and $\operatorname{im}(F)=M$. Our main result is that $M=\operatorname{im}(F)$ is closed in $\left[\mathscr{E}^{m}(\Omega)\right]^{p}$ if and only if the rank of $F(x)=\left(f_{i j}(x)\right)_{1 \leqq i \leqq p, 1 \leqq j \leqq q}$ is constant for $x \in \Omega$.

The sufficiency of the condition that $\operatorname{rank}(F(x))$ be constant is a direct

Received by the editors January 22, 1971.

AMS 1970 subject classifications. Primary 46E25, 46F10.

Key words and phrases. Spaces of differentiable functions, modules of differentiable functions, finitely generated submodules, spaces of distributions, division of distributions.

(C) American Mathematical Society 1972 
consequence of Malgrange's characterization of the closure of a submodule of differentiable functions [1, Corollary 1.6, p. 25]. For continuous functions, that is, when $m=0$, the necessity of the condition that $\operatorname{rank}(F(x))$ be constant can be shown using the open mapping theorem (see [4, Theorem 3.1]). However, in general, a different method of proof is required. Assuming that $M=\operatorname{im}(F)$ is closed in $\left[\mathscr{E}^{m}(\Omega)\right]^{p}$, we have im $\left(F^{\prime}\right)=[\operatorname{ker}(F)]^{\perp}$ where $F^{\prime}:\left[\mathscr{E}^{\prime m}(\Omega)\right]^{p} \rightarrow\left[\mathscr{E}^{\prime m}(\Omega)\right]^{q}$ is the transpose of $F$. Assuming that $\operatorname{rank}(F(x))$ is not constant on $\Omega$, we find a $q$-tuple of distributions

$$
\left(T_{1}, \cdots, T_{q}\right) \in[\operatorname{ker}(F)]^{\perp}
$$

such that for some $j, 1 \leqq j \leqq q$, the support of $T_{j}$ consists of a single point where the rank of $F$ is "changing" and the order of $T_{j}$ is "large". Therefore $F^{\prime}\left(S_{1}, \cdots, S_{p}\right)=\left(T_{1}, \cdots, T_{q}\right)$ for some $\left(S_{1}, \cdots, S_{p}\right) \in\left[\mathscr{E}^{\prime \prime m}(\Omega)\right]^{p}$ which is impossible because the order of $T_{j}$ is too large.

We first prove a lemma establishing the existence of a $\left(T_{1}, \cdots, T_{q}\right) \in$ $[\operatorname{ker}(F)]^{\perp}$ with the appropriate properties.

Lemma 2.1. Suppose $F=\left(f_{i j}\right)_{1 \leqq i \leqq p, 1 \leqq j \leqq q}, f_{i j} \in \mathscr{E}^{m}(\Omega)$. Suppose

$$
a \in \operatorname{bd}\{x \in \Omega: \operatorname{rank}(F(x))=r\} \quad \text { where } r=\max \{\operatorname{rank}(F(x)): x \in \Omega\} .
$$

Let $s=\operatorname{rank}(F(a))$. Then there exists $\left(T_{1}, \cdots, T_{q}\right) \in[\operatorname{ker}(F)]^{\perp}$ such that:

(1) for some $j_{1}, \cdots, j_{s}, T_{j_{1}}=\cdots=T_{j_{s}}=0$ and the $j_{1}, \cdots, j_{s}$ columns of $F(a)$ are linearly independent;

(2) for some $j_{s+1}, \operatorname{supp}\left(T_{j_{s+1}}\right)=\{a\}$ and $\operatorname{order}\left(T_{j_{s+1}}\right)=m-k$ where $k$ is the largest integer $\leqq m$ such that the determinant of every $(s+1) \times(s+1)$ submatrix of $F$ with rank $s$ at a vanishes at a together with all derivatives of order $\leqq k$.

Proof. If $s=0$, the lemma asserts that there exists $\left(T_{1}, \cdots, T_{q}\right) \in$ $[\operatorname{ker}(F)]^{\perp}$ such that for some $j, 1 \leqq j \leqq q, \operatorname{supp}\left(T_{j}\right)=\{a\}$ and $\operatorname{order}\left(T_{j}\right)=$ $m-k$ where $k$ is the largest integer $\leqq m$ such that every $f_{i j}$ vanishes at $a$ together with all derivatives of order $\leqq k$. Since the proof for $s=0$ is similar to the proof for $s>0$, we assume $s>0$.

Let $k$ be as in (2). If $k=m$, then there exists an $(s+1) \times(s+1)$ submatrix of $F$ with rank $s$ at $a$ which is nonsingular at every point of a sequence $\left\{x_{l}\right\}$ in $\Omega$ with $x_{l} \rightarrow a$ since $a \in \operatorname{bd}\{x \in \Omega: \operatorname{rank}(F(x))=r\}$. If $k<m$, then there exists an $(s+1) \times(s+1)$ submatrix of $F$ with rank $s$ at $a$ whose determinant has a zero of order exactly $k$ at $a$ by our choice of $k$. In each case, for simplicity suppose that $\left(f_{i j}\right)_{1 \leqq i, j \leqq s+1}$ is the $(s+1) \times(s+1)$ submatrix of $F$ and the first $s$ columns of $\left(f_{i j}(a)\right)_{1 \leqq i, j \leqq s+1}$ are linearly independent. 
Consider $\left(g_{1}, \cdots, g_{q}\right) \in \operatorname{ker}(F)$. Then

$$
\begin{aligned}
& f_{11} g_{1}+\cdots+f_{1 q} g_{q}=0, \\
& f_{(s+1) 1} g_{1}+\cdots+f_{(s+1) q} g_{q}=0 .
\end{aligned}
$$

For $1 \leqq i \leqq s+1$, multiply the $i$ th row of this system of equations by the cofactor of $f_{i(s+1)}$ in $\left(f_{i j}\right)_{1 \leqq i, j \leqq s+1}$. Adding the equations thus obtained, we find that $d_{1} g_{1}+\cdots+d_{q} g_{q}=0$ where for $1 \leqq j \leqq q$,

$$
d_{j}=\operatorname{det}\left(\begin{array}{cccc}
f_{11} & \cdots & f_{1 s} & f_{1 j} \\
\cdot & & \cdot & \cdot \\
\cdot & & \cdot & \cdot \\
\cdot & & \cdot & \cdot \\
f_{(s+1) 1} & \cdots & f_{(s+1) s} & f_{(s+1) j}
\end{array}\right) .
$$

Clearly $d_{1}=\cdots=d_{s}=0$ so $d_{s+1} g_{s+1}+\cdots+d_{q} g_{q}=0$ for all $\left(g_{1}, \cdots, g_{q}\right) \in$ $\operatorname{ker}(F)$.

Define $D:\left[\mathscr{E}^{m}(\Omega)\right]^{q-s_{\rightarrow} \rightarrow \mathscr{E}^{m}}(\Omega)$ by $D\left(h_{1}, \cdots, h_{q-s}\right)=d_{s+1} h_{1}+\cdots+d_{q} h_{q-s}$. Then whenever $\left(g_{1}, \cdots, g_{q}\right) \in \operatorname{ker}(F)$, we have $\left(g_{s+1}, \cdots, g_{q}\right) \in \operatorname{ker}(D)$. If $k=m$, then $d_{s+1}$ is nonzero at every point of a sequence $\left\{x_{l}\right\}$ in $\Omega$ with $x_{l} \rightarrow a$, while if $k<m$, then each $d_{j}, s+1 \leqq j \leqq q$, has a zero of order at least $k$ at $a$ and $d_{s+1}$ has a zero of order exactly $k$ at $a$. Applying Lemma 3.1 of [2, p. 215] if $k=m$ and Lemma 3.2 of [2, p. 215] if $k<m$, we find that there exists $\left(T_{1}, \cdots, T_{q-s}\right) \in[\operatorname{ker}(D)]^{\perp}$ such that for some $j, 1 \leqq j \leqq q-s$, $\operatorname{supp}\left(T_{j}\right)=\{a\}$ and $\operatorname{order}\left(T_{j}\right)=m-k$. Then $\left(0, \cdots, 0, T_{1}, \cdots, T_{q-s}\right) \in$ $[\operatorname{ker}(F)]^{\perp}$ and satisfies (1) and (2).

THEOREM 2.1. Suppose $F=\left(f_{i j}\right)_{1 \leqq i \leqq p, 1 \leqq j \leqq q}, f_{i j} \in \mathscr{E}^{m}(\Omega)$. The finitely generated submodule $M=\operatorname{im}(F)$ of $\left[\mathscr{E}^{m}(\Omega)\right]^{p}$ is closed in $\left[\mathscr{E}^{m}(\Omega)\right]^{p}$ if and only if the rank of $F(x)=\left(f_{i j}(x)\right)_{1 \leqq i \leqq p, 1 \leqq j \leqq q}$ is constant for $x \in \Omega$.

Proof. Suppose $M=\operatorname{im}(F)$ is closed in $\left[\mathscr{E}^{m}(\Omega)\right]^{p}$ but $\operatorname{rank}(F(x))$ is not constant on $\Omega$. Let $r=\max \{\operatorname{rank}(F(x)): x \in \Omega\}$. Since $\Omega$ is connected, there exists $a=\left(a_{1}, \cdots, a_{n}\right) \in \operatorname{bd}\{x \in \Omega: \operatorname{rank}(F(x))=r\}$. Let $s=\operatorname{rank}(F(a))$. By Lemma 2.1, there exists $\left(T_{1}, \cdots, T_{q}\right) \in[\operatorname{ker}(F)]^{\perp}$ satisfying (1) and (2) where we assume for the sake of simplicity that $j_{1}=1, \cdots, j_{s}=s, j_{s+1}=s+1$.

Since $\operatorname{im}(F)$ is closed in $\left[\mathscr{E}^{m}(\Omega)\right]^{p}$, the closed range theorem for Fréchet spaces implies that $\operatorname{im}\left(F^{\prime}\right)=[\operatorname{ker}(F)]^{\perp}$ where $F^{\prime}:\left[\mathscr{E}^{\prime m}(\Omega)\right]^{p} \rightarrow\left[\mathscr{E}^{\prime m}(\Omega)\right]^{q}$ is the transpose of $F$. Hence there exists $\left(S_{1}, \cdots, S_{p}\right) \in\left[\mathscr{E}^{\prime m}(\Omega)\right]^{p}$ with 
$F^{\prime}\left(S_{1}, \cdots, S_{p}\right)=\left(T_{1}, \cdots, T_{q}\right)$. Letting $f_{i j}^{\prime}=f_{j i}$ for $1 \leqq i \leqq q, 1 \leqq j \leqq p$, we have

$$
\begin{aligned}
& f_{11}^{\prime} S_{1}+\cdots+f_{1 p}^{\prime} S_{p}=T_{1}=0 \\
& f_{s 1}^{\prime} S_{1}+\cdots+f_{s p}^{\prime} S_{p}=T_{s}=0, \\
& f_{(s+1) 1}^{\prime} S_{1}+\cdots+f_{(s+1) p}^{\prime} S_{p}=T_{s+1} \text {. }
\end{aligned}
$$

By (1), the columns of $\left(f_{i j}(a)\right)_{1 \leqq i \leqq p, 1 \leqq j \leqq s}$ are linearly independent so $s$ rows of $\left(f_{i j}(a)\right)_{1 \leqq i \leqq p, 1 \leqq j \leqq s}$ are linearly independent, say for simplicity the first $s$ rows. Then $\operatorname{det}\left(f_{i j}^{\prime}(a)\right)_{1 \leqq i, j \leqq s}=\operatorname{det}\left(f_{i j}(a)\right)_{1 \leqq i, j \leqq s} \neq 0$. For $1 \leqq i \leqq s+1$, multiply the $i$ th row in the above system of equations by the cofactor of $f_{i(s+1)}^{\prime}$ in $\left(f_{i j}^{\prime}\right)_{1 \leqq i, j \leqq s+1}$. Adding the equations thus obtained we find that

$$
g_{1} S_{1}+\cdots+g_{p} S_{p}=\operatorname{det}\left(f_{i j}^{\prime}\right)_{1 \leqq i, j \leqq s} T_{s+1},
$$

where for $1 \leqq j \leqq p$,

$$
g_{j}=\operatorname{det}\left(\begin{array}{cccc}
f_{11}^{\prime} & \cdots & f_{1 s}^{\prime} & f_{1 j}^{\prime} \\
\cdot & & \cdot & \cdot \\
\cdot & & \cdot & \cdot \\
\cdot & & \cdot & \cdot \\
f_{(s+1) 1}^{\prime} & \cdots & f_{(s+1) s}^{\prime} & f_{(s+1) j}^{\prime}
\end{array}\right) .
$$

By our choice of $k$ in (2), the functions $g_{1}, \cdots, g_{p}$ each vanish at $a$ together with all derivatives of order $\leqq k$. And by $(2), \operatorname{supp}(T)=\{a\}$ and $\operatorname{order}(T)=$ $m-k$ where $T=\operatorname{det}\left(f_{i j}^{\prime}\right)_{1 \leqq i, j \leqq s} T_{s+1}$ since $\operatorname{det}\left(f_{i j}^{\prime}(a)\right)_{1 \leqq i, j \leqq s} \neq 0$.

The equation $g_{1} S_{1}+\cdots+g_{p} S_{p}=T$ together with Malgrange's characterization of the closure of a submodule of differentiable functions leads to a contradiction. To see this, define $G: \mathscr{E}^{m}(\Omega) \rightarrow\left[\mathscr{E}^{m}(\Omega)\right]^{p}$ by $G(h)=\left(g_{1} h\right.$, $\left.\cdots, g_{p} h\right)$ and let $G^{\prime}:\left[\mathscr{E}^{\prime m}(\Omega)\right]^{p} \rightarrow \mathscr{E}^{\prime m}(\Omega)$ be the transpose of $G$. Then $G^{\prime}\left(S_{1}, \cdots, S_{p}\right)=g_{1} S_{1}+\cdots+g_{p} S_{p}=T$. Define $f \in \mathscr{E}^{m}(\Omega)$ by $f\left(x_{1}, \cdots, x_{n}\right)=$ $\left(x_{1}-a_{1}\right)^{l}+\cdots+\left(x_{n}-a_{n}\right)^{l}$ where $l$ is an even integer $>m$. Define $g \in \mathscr{E}^{m}(\Omega)$ by $g\left(x_{1}, \cdots, x_{n}\right)=\left(x_{1}-a_{1}\right)^{\alpha_{1}} \cdots\left(x_{n}-a_{n}\right)^{\alpha_{n}}$ where $\alpha_{1}, \cdots, \alpha_{n}$ are chosen so that $\alpha_{1}+\cdots+\alpha_{n}=m-k$ and $\partial_{1}^{\alpha_{1}} \cdots \partial_{n}^{\alpha_{n}} \delta_{a}$ appears with a nonzero coefficient in $T$.

Then $G^{\prime}\left(f S_{1}, \cdots, f S_{p}\right)=f T=0$ and thus $\left(f S_{1}, \cdots, f S_{p}\right) \in \operatorname{ker}\left(G^{\prime}\right)=N^{-L}$ where $N=\operatorname{im}(G)$ is a submodule of $\left[\mathscr{E}^{m}(\Omega)\right]^{p}$. Hence $\left(S_{1}, \cdots, S_{p}\right) \in(f N)^{\perp}$. For $x \in \Omega$, let $T_{x}^{m}$ be the natural mapping of $\left[\mathscr{E}^{m}(\Omega)\right]^{p}$ onto $\left[\mathscr{E}^{m}(\Omega)\right]^{p} /\left[J_{x}^{m}\right]^{p}$ where $J_{x}^{m}$ is the ideal in $\mathscr{E}^{m}(\Omega)$ consisting of all functions which vanish at $x$ together with all derivatives of order $\leqq m$. Then $T_{a}^{m}(f N)$ and $T_{a}^{m}(g N)$ are both the zero submodule in $\left[\mathscr{E}^{m}(\Omega)\right]^{p} /\left[J_{a}^{m}\right]^{p}$. And for $x \in \Omega, x \neq a$, we have 
$T_{x}^{m}(g N) \subset T_{x}^{m}(f N)$ since $f(x) \neq 0$. By Malgrange's theorem [1, Corollary 1.6, p. 25], we conclude that $\operatorname{cl}(g N) \subset \operatorname{cl}(f N)$ and hence $(f N)^{\perp} \subset(g N)^{\perp}$. Therefore $\left(S_{1}, \cdots, S_{p}\right) \in(g N)^{\perp}$ and thus $\left(g S_{1}, \cdots, g S_{p}\right) \in N^{\perp}=\operatorname{ker}\left(G^{\prime}\right)$. Hence $0=G^{\prime}\left(g S_{1}, \cdots, g S_{p}\right)=g T$. But $g T \neq 0$ since $\partial_{1}^{\alpha_{1}} \cdots \partial_{n}^{\alpha_{n}} \delta_{a}$ appears with a nonzero coefficient in $T$. This contradiction completes the proof of the necessity that $\operatorname{rank}(F(x))$ be constant on $\Omega$.

Conversely, suppose $\operatorname{rank}(F(x))=r$ for all $x \in \Omega$. Consider $\left(h_{1}, \cdots, h_{p}\right) \in$ $\operatorname{cl}(M)$. Then Malgrange's theorem says that $\left(h_{1}, \cdots, h_{p}\right)$ is pointwise in $M$, that is, $T_{x}^{m}\left(h_{1}, \cdots, h_{p}\right) \in T_{x}^{m}(M)$ for all $x \in \Omega$. This fact enables us to find $\left(g_{1}, \cdots, g_{q}\right) \in\left[\mathscr{E}^{m}(\Omega)\right]^{q}$ with $F\left(g_{1}, \cdots, g_{q}\right)=\left(h_{1}, \cdots, h_{p}\right)$ and thus $\left(h_{1}, \cdots, h_{p}\right) \in \operatorname{im}(F)=M$.

Since the case $r=0$ is trivial, we assume $r>0$. There exists a locally finite open covering $\left\{\Omega_{\alpha}\right\}$ of $\Omega$ with the property that for each $\Omega_{\alpha}$ there exists an $r \times r$ submatrix of $F$ which is nonsingular at every $x \in \Omega_{\alpha}$. Using a partition of unity argument, it is easy to see that it suffices to find $\left(g_{1}, \cdots, g_{q}\right) \in\left[\mathscr{E}^{m}\left(\Omega_{\alpha}\right)\right]^{p}$ with $F\left(g_{1}, \cdots, g_{q}\right)=\left(h_{1}, \cdots, h_{p}\right)$ in $\Omega_{\alpha}$ for each $\alpha$.

Suppose $\Omega_{\alpha}$ is a member of the open covering. Then some $r \times r$ submatrix of $F$ is nonsingular at every $x \in \Omega_{\alpha}$, say for $\operatorname{simplicity}\left(f_{i j}\right)_{1 \leqq i, j \leqq r}$. Then the system of equations

$$
\begin{aligned}
& f_{11} g_{1}+\cdots+f_{1 r} g_{r}=h_{1}, \\
& f_{r 1} g_{1}+\cdots+f_{r r} g_{r}=h_{r},
\end{aligned}
$$

can be uniquely solved in $\Omega_{\alpha}$ for functions $g_{1}, \cdots, g_{r} \in \mathscr{E}^{m}\left(\Omega_{\alpha}\right)$. Consider $\left(g_{1}, \cdots, g_{q}\right) \in\left[\mathscr{E}^{m}\left(\Omega_{\alpha}\right)\right]^{q}$ where $g_{j}=0$ for $r<j \leqq q$.

We claim that $F\left(g_{1}, \cdots, g_{q}\right)=\left(h_{1}, \cdots, h_{p}\right)$ in $\Omega_{\alpha}$, that is, $f_{i 1} g_{1}+\cdots$ $+f_{i q} g_{q}=h_{i}$ in $\Omega_{\alpha}$ for $1 \leqq i \leqq p$. Since in $\Omega_{\alpha}$ for $1 \leqq i \leqq p$ we have

$$
h_{i}-\sum_{j=1}^{q} f_{i j} g_{j}=\operatorname{det}\left(\begin{array}{cccc}
f_{11} & \cdots & f_{1 r} & h_{1} \\
\cdot & & \cdot & \cdot \\
\cdot & & \cdot & \cdot \\
\cdot & & \cdot & \cdot \\
f_{r 1} & \cdots & f_{r r} & h_{r} \\
f_{i 1} & \cdots & f_{i r} & h_{i}
\end{array}\right) / \operatorname{det}\left(f_{i j}\right)_{1 \leqq i, j \leqq r},
$$

it suffices to prove that the determinant in the numerator is zero in $\Omega_{\alpha}$ for $1 \leqq i \leqq p$. 
Consider $x \in \Omega_{\alpha}$. Since $\left(h_{1}, \cdots, h_{p}\right)$ is pointwise in $M=\operatorname{im}(F)$, the column vector $\left(h_{i}(x)\right)_{1 \leqq i \leqq p}$ is linearly dependent on the columns of the matrix $\left(f_{i j}(x)\right)_{1 \leqq i \leqq p, 1 \leqq j \leqq q}$. Since $\left(f_{i j}(x)\right)_{1 \leqq i, j \leqq r}$ is nonsingular and $\operatorname{rank}(F(x))=r$, the last $q-r$ columns of $\left(f_{i j}(x)\right)_{1 \leqq i \leqq p, 1 \leqq j \leqq q}$ are linearly dependent on the first $r$ columns. Hence the column vector $\left(h_{i}(x)\right)_{1 \leqq i \leqq p}$ is linearly dependent on the columns of the matrix $\left(f_{i j}(x)\right)_{1 \leqq i \leqq p, 1 \leqq j \leqq r}$. Therefore

$$
\operatorname{det}\left(\begin{array}{cccc}
f_{11}(x) & \cdots & f_{1 r}(x) & h_{1}(x) \\
\cdot & & \cdot & \cdot \\
\cdot & & \cdot & \cdot \\
\cdot & & \cdot & \cdot \\
f_{r 1}(x) & \cdots & f_{r r}(x) & h_{r}(x) \\
f_{i 1}(x) & \cdots & f_{i r}(x) & h_{i}(x)
\end{array}\right)=0
$$

for $1 \leqq i \leqq p$ which completes the proof of Theorem 2.1.

Corollary 2.1. Suppose $f_{1}, \cdots, f_{q} \in \mathscr{E}^{m}(\Omega)$. The ideal I generated by $f_{1}, \cdots, f_{q}$ in $\mathscr{E}^{m}(\Omega)$ is closed in $\mathscr{E}^{m}(\Omega)$ if and only if $f_{1}^{2}+\cdots+f_{q}^{2}$ never vanishes or $f_{1}^{2}+\cdots+f_{a}^{2}$ is identically zero.

Proof. Let $F=\left(f_{j}\right)_{1 \leqq j \leqq x}$. Then $\operatorname{rank}(F(x))=0$ for all $x \in \Omega$ if and only if $f_{1}^{2}+\cdots+f_{a}^{2}$ is identically zero in $\Omega$. And $\operatorname{rank}(F(x))=1$ for all $x \in \Omega$ if and only if $f_{1}^{2}+\cdots+f_{q}^{2}$ is never zero in $\Omega$.

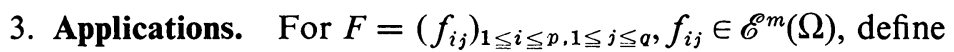
$F:\left[\mathscr{D}^{\prime m}(\Omega)\right]^{q} \rightarrow\left[\mathscr{D}^{\prime m}(\Omega)\right]^{p}$ by

$$
F\left(S_{1}, \cdots, S_{q}\right)=\left(\sum_{j=1}^{q} f_{1 j} S_{j}, \cdots, \sum_{j=1}^{q} f_{p j} S_{j}\right) .
$$

Let $F_{c}:\left[\mathscr{E}^{\prime m}(\Omega)\right]^{q} \rightarrow\left[\mathscr{E}^{\prime m}(\Omega)\right]^{p}$ be the restriction of $F$ to $\left[\mathscr{E}^{\prime m}(\Omega)\right]^{q}$. Let $F^{\prime}:\left[\mathscr{D}^{m}(\Omega)\right]^{p} \rightarrow\left[\mathscr{D}^{m}(\Omega)\right]^{q}$ (respectively $F_{c}^{\prime}:\left[\mathscr{E}^{m}(\Omega)\right]^{p} \rightarrow\left[\mathscr{E}^{m}(\Omega)\right]^{q}$ ) be the transpose of $F$ (respectively $F_{c}$ ).

Consider $F\left(S_{1}, \cdots, S_{q}\right)=\left(T_{1}, \cdots, T_{p}\right)$, that is, the system of division problems $f_{i 1} S_{1}+\cdots+f_{i q} S_{q}=T_{i}$ for $1 \leqq i \leqq p$, where $\left(T_{1}, \cdots, T_{p}\right) \in\left[\mathscr{D}^{\prime m}(\Omega)\right]^{p}$. In order that there exist a solution $\left(S_{1}, \cdots, S_{q}\right) \in\left[\mathscr{D}^{\prime m}(\Omega)\right]^{q}$, it is necessary that $\left(T_{1}, \cdots, T_{p}\right) \in\left[\operatorname{ker}\left(F^{\prime}\right)\right]^{\perp}$ since $\operatorname{im}(F) \subset\left[\operatorname{ker}\left(F^{\prime}\right)\right]^{\perp}$. Equivalently, it is necessary that every relation between the rows of $\left(f_{i j}\right)_{1 \leqq i \leqq p, 1 \leqq j \leqq q}$ be a relation between $\left(T_{1}, \cdots, T_{p}\right)$, that is, if $\psi_{1} \mathbf{f}_{1}+\cdots+\psi_{p} \mathbf{f}_{p}=(0, \cdots, 0)$ where $\mathbf{f}_{i}=\left(f_{i 1}, \cdots, f_{i q}\right)$ and $\psi_{i} \in \mathscr{D}^{m}(\Omega)$ for $1 \leqq i \leqq p$, then $\psi_{1} T_{1}+\cdots+$ $\psi_{p} T_{p}=0$. We are interested in those cases where for every $\left(T_{1}, \cdots, T_{p}\right) \in$ $\left[\operatorname{ker}\left(F^{\prime}\right)\right]^{\perp}$ there exists $\left(S_{1}, \cdots, S_{q}\right) \in\left[\mathscr{D}^{\prime m}(\Omega)\right]^{q}$ satisfying $F\left(S_{1}, \cdots, S_{q}\right)=$ $\left(T_{1}, \cdots, T_{p}\right)$ and thus are led to the following definition. 
Definition. $\left[\mathscr{D}^{\prime m}(\Omega)\right]^{p}$ is divisible by $F=\left(f_{i j}\right)_{1 \leqq i \leqq p, 1 \leqq j \leq q}$ if for every $\left(T_{1}, \cdots, T_{p}\right) \in\left[\operatorname{ker}\left(F^{\prime}\right)\right]^{\perp}$ there exists $\left(S_{1}, \cdots, S_{q}\right) \in\left[\mathscr{D}^{\prime m}(\Omega)\right]^{q}$ with $F\left(S_{1}, \cdots, S_{q}\right)=\left(T_{1}, \cdots, T_{p}\right)$.

Then $\left[\mathscr{D}^{\prime m}(\Omega)\right]^{p}$ is divisible by $F=\left(f_{i j}\right)_{1 \leqq i \leqq p, 1 \leqq j \leqq q}$ if and only if $\operatorname{im}(F)=$ $\left[\operatorname{ker}\left(F^{\prime}\right)\right]^{\perp}$. A simple partition of unity argument establishes that $\operatorname{im}(F)=$ $\left[\operatorname{ker}\left(F^{\prime}\right)\right]^{\perp}$ if and only if $\operatorname{im}\left(F_{c}\right)=\left[\operatorname{ker}\left(F_{c}^{\prime}\right)\right]^{\perp}$. The closed range theorem for Fréchet spaces now provides the link between systems of division problems for distributions and finitely generated submodules of differentiable functions. For it says that $\operatorname{im}\left(F_{c}\right)=\left[\operatorname{ker}\left(F_{c}^{\prime}\right)\right]^{\perp}$ if and only if $\operatorname{im}\left(F_{c}^{\prime}\right)$, which is the submodule generated by $\mathbf{f}_{1}, \cdots, \mathbf{f}_{p}$ in $\left[\mathscr{E}^{m}(\Omega)\right]^{q}$ where $\mathbf{f}_{i}=\left(f_{i 1}, \cdots, f_{i q}\right)$ for $1 \leqq i \leqq p$, is closed in $\left[\mathscr{E}^{m}(\Omega)\right]^{q}$. Therefore Theorem 2.1 gives the following result.

THEOREM 3.1. $\left[\mathscr{D}^{\prime m}(\Omega)\right]^{p}$ is divisible by $F=\left(f_{i j}\right)_{1 \leqq i \leqq p, 1 \leqq j \leqq q}$ if and only if $\operatorname{rank}(F(x))$ is constant for $x \in \Omega$.

Corollary 3.1. $\operatorname{im}(F)=\left[\mathscr{D}^{\prime m}(\Omega)\right]^{p}$ where $F=\left(f_{i j}\right)_{1 \leqq i \leqq p, 1 \leqq j \leqq q}$ if and only if $\operatorname{rank}(F(x))=p$ for all $x \in \Omega$.

Proof. $\operatorname{im}(F)=\left[\mathscr{D}^{\prime m}(\Omega)\right]^{p}$ if and only if $\left[\mathscr{D}^{\prime m}(\Omega)\right]^{p}$ is divisible by $F$ and $F^{\prime}:\left[\mathscr{D}^{m}(\Omega)\right]^{p} \rightarrow\left[\mathscr{D}^{m}(\Omega)\right]^{q}$ is one-to-one. It can be shown that $F^{\prime}:\left[\mathscr{D}^{m}(\Omega)\right]^{p} \rightarrow\left[\mathscr{D}^{m}(\Omega)\right]^{q}$ is one-to-one if and only if the set of $x \in \Omega$ for which $\operatorname{rank}(F(x))=p$ is dense in $\Omega$ (see [3, Lemma 3.1]). The corollary now follows immediately from Theorem 3.1.

\section{BIBLIOGRAPHY}

1. B. Malgrange, Ideals of differentiable functions, Tata Inst. Fund. Res. Studies in Math., no. 3, Tata Inst. of Fundamental Research, Bombay; Oxford Univ. Press, London, 1966. MR 35 \#3446.

2. B. Roth, Finitely generated ideals of differentiable functions, Trans. Amer. Math. Soc. 150 (1970), 213-225. MR 41 \# 7415.

3. - Systems of division problems for distributions, Trans. Amer. Math. Soc. 155 (1971), 493-504.

4. - Submodules of $C(X) \times \cdots \times C(X)$, Proc. Amer. Math. Soc. 32 (1972), $543-548$.

Department of Mathematics, University of Wyoming, Laramie, Wyoming 82070 